\title{
Periodic Solutions and Exponential Stability of a Class of Neural Networks with Time-Varying Delays
}

\author{
Yingxin $\mathbf{G u o}^{\mathbf{1}}$ and Mingzhi Xue ${ }^{2}$ \\ ${ }^{1}$ Department of Mathematics, Qufu Normal University, Qufu 273165, Shandong, China \\ ${ }^{2}$ Department of Mathematics, Shangqiu Normal University, Shangqiu 476000, Henan, China
}

Correspondence should be addressed to Mingzhi Xue, whl2762@163.com

Received 8 March 2009; Accepted 17 June 2009

Recommended by Guang Zhang

Employing fixed point theorem, we make a further investigation of a class of neural networks with delays in this paper. A family of sufficient conditions is given for checking global exponential stability. These results have important leading significance in the design and applications of globally stable neural networks with delays. Our results extend and improve some earlier publications.

Copyright (C) 2009 Y. Guo and M. Xue. This is an open access article distributed under the Creative Commons Attribution License, which permits unrestricted use, distribution, and reproduction in any medium, provided the original work is properly cited.

\section{Introduction}

The stability of dynamical neural networks with time delay which have been used in many applications such as optimization, control, and image processing has received much attention recently (see, e.g., [1-15]). Particularly, the authors $[3,8,9,14,16]$ have studied the stability of neural networks with time-varying delays.

As pointed out in [8], Global dissipativity is also an important concept in dynamical neural networks. The concept of global dissipativity in dynamical systems is a more general concept, and it has found applications in areas such as stability theory, chaos and synchronization theory, system norm estimation, and robust control [8]. Global dissipativity of several classes of neural networks was discussed, and some sufficient conditions for the global dissipativity of neural networks with constant delays are derived in [8].

In this paper, without assuming the boundedness, monotonicity, and differentiability of activation functions, we consider the following delay differential equations: 


$$
\begin{aligned}
x_{i}^{\prime}(t)= & -d_{i}(t) x_{i}(t)+\sum_{j=1}^{n} a_{i j}(t) f_{j}\left(x_{j}(t)\right)+\sum_{j=1}^{n} b_{i j}(t) f_{j}\left(x_{j}\left(t-\tau_{i j}(t)\right)\right) \\
& +\sum_{j=1}^{n} c_{i j}(t) \int_{-\infty}^{t} H_{i j}(t-s) f_{j}\left(x_{j}(s)\right) d s+J_{i}(t), \quad i=1,2, \ldots, n,
\end{aligned}
$$

where $n$ denotes the number of the neurons in the network, $x_{i}(t)$ is the state of the $i$ th neuron at time $t, x(t)=\left[x_{1}(t), x_{2}(t), \ldots, x_{n}(t)\right]^{T} \in R^{n}, f(x(t))=\left[f_{1}\left(x_{1}(t)\right), f_{2}\left(x_{2}(t)\right), \ldots, f_{n}\left(x_{n}(t)\right)\right]^{T} \in$ $R^{n}$ denote the activation functions of the $j$ th neuron at time $t$, and the kernels $H_{i j}:[0,+\infty) \rightarrow$ $[0,+\infty)$ are piece continuous functions with $\int_{0}^{+\infty} H_{i j}(s) d s=h_{i j}<\infty$ for $i, j=1,2, \ldots, n$. Moreover, we consider model (1.1) with $\tau_{i j}(t), d_{i}(t), a_{i j}(t), b_{i j}(t), c_{i j}(t)$, and $J_{i}(t)$ satisfying the following assumptions:

(A1) the time delays $\tau_{i j}(t) \in C(R,[0, \infty))$ are periodic functions with a common period $\omega(>0)$ for $i, j=1,2, \ldots, n$;

(A2) $c_{i j}(t) \in C(R,[0, \infty)), a_{i j}(t), b_{i j}(t), c_{i j}(t), J_{i}(t) \in C(R, R)$ are periodic functions with a common period $\omega(>0)$ and $f_{i} \in C(R, R), i, j=1,2, \ldots, n$.

The organization of this paper is as follows. In Section 2, problem formulation and preliminaries are given. In Section 3, some new results are given to ascertain the global robust dissipativity of the neural networks with time-varying delays. Section 4 gives an example to illustrate the effectiveness of our results.

\section{Preliminaries and Lemmas}

For the sake of convenience, two of the standing assumptions are formulated below as follows.

(A3) $\left|f_{j}(u)\right| \leq p_{j}|u|+q_{j}$ for all $u \in R, j=1,2, \ldots, n$, where $p_{j}, q_{j}$ are nonnegative constants.

(A4) There exist nonnegative constants $p_{j}, j=1,2, \ldots, n$, such that $\left|f_{j}(u)-f_{j}(v)\right| \leq p_{j} \mid u-$ $v \mid$ for any $u, v \in R$.

Let

$$
\tau=\max _{1 \leq i, j \leq n} \sup _{t \geq 0}\left\{\tau_{i j}(t)\right\}
$$

The initial conditions associated with system (1.1) are of the form

$$
x_{i}(s)=\phi_{i}(s), \quad s \in[-\tau, 0], i=1,2, \ldots, n,
$$

in which $\phi_{i}(s)$ is continuous for $s \in[-\tau, 0]$.

For continuous functions $\phi_{i}$ defined on $[-\tau, 0], i=1,2, \ldots, n$, we set $\phi=$ $\left(\phi_{1}, \phi_{2}, \ldots, \phi_{n}\right)^{T}$. If $x^{0}=\left(x_{1}^{0}, x_{1}^{0}, \ldots, x_{n}^{0}\right)^{T}$ is an equilibrium of system (1.1), then we denote

$$
\left\|\phi-x^{0}\right\|=\sum_{i=1}^{n}\left(\sup _{-\tau \leq t \leq 0}\left|\phi_{i}(t)-x_{i}^{0}\right|\right) .
$$


Definition 2.1. The equilibrium $x^{0}=\left(x_{1}^{0}, x_{1}^{0}, \ldots, x_{n}^{0}\right)^{T}$ is said to be globally exponentially stable, if there exist constants $\lambda>0$ and $m \geq 1$ such that for any solution $x(t)=$ $\left(x_{1}(t), x_{2}(t), \ldots, x_{n}(t)\right)^{T}$ of $(1.1)$, we have

$$
\left|x_{i}(t)-x_{i}^{0}\right| \leq m\left\|\phi-x^{0}\right\| e^{-\lambda t}
$$

for $t \geq 0$, where $\lambda$ is called to be globally exponentially convergent rate.

Lemma 2.2 ([17]). If $\rho(K)<1$ for matrix $K=\left(k_{i j}\right)_{n \times n} \geq 0$, then $(E-K)^{-1} \geq 0$, where E denotes the identity matrix of size $n$.

\section{Periodic Solutions and Exponential Stability}

We will use the coincidence degree theory to obtain the existence of a $\omega$-periodic solution to systems (1.1). For the sake of convenience, we briefly summarize the theory as follows.

Let $X$ and $Z$ be normed spaces, and let $L: \operatorname{Dom} L \subset X \mapsto Z$ be a linear mapping and be a continuous mapping. The mapping $L$ will be called a Fredholm mapping of index zero if $\operatorname{dimKer} L=\operatorname{codimIm} L<\infty$ and $\operatorname{Im} L$ is closed in $Z$. If $L$ is a Fredholm mapping of index zero, then there exist continuous projectors $P: X \mapsto X$ and $Q: Z \mapsto Z$ such that $\operatorname{Im} P=\operatorname{Ker} L$ and $\operatorname{Im} L=\operatorname{Ker} Q=\operatorname{Im}(I-Q)$. It follows that $L \mid \operatorname{Dom} L \cap \operatorname{Ker} P:(I-P) X \mapsto \operatorname{Im} L$ is invertible. We denote the inverse of this map by $K_{p}$. If $\Omega$ is a bounded open subset of $X$, the mapping $N$ is called $L$-compact on $\bar{\Omega}$ if $Q N(\bar{\Omega})$ is bounded and $K_{p}(I-Q) N: \bar{\Omega} \mapsto X$ is compact. Because $\operatorname{Im} Q$ is isomorphic to $\operatorname{Ker} L$, there exists an isomorphism $J: \operatorname{Im} Q \mapsto \operatorname{Ker} L$.

Let $\Omega \subset R^{n}$ be open and bounded, $f \in C^{1}\left(\Omega, R^{n}\right) \cap C\left(\bar{\Omega}, R^{n}\right)$ and $y \in R^{n} \backslash f\left(\partial \Omega \cup S_{f}\right)$, that is, $y$ is a regular value of $f$. Here, $S_{f}=\left\{x \in \Omega: J_{f}(x)=0\right\}$, the critical set of $f$, and $J_{f}$ is the Jacobian of $f$ at $x$. Then the degree $\operatorname{deg}\{f, \Omega, y\}$ is defined by

$$
\operatorname{deg}\{f, \Omega, y\}=\sum_{x \in f^{-1}(y)} \operatorname{sgn} J_{f}(x)
$$

with the agreement that the above sum is zero if $f^{-1}(y)=\emptyset$. For more details about the degree theory, we refer to the book of Deimling [18].

Lemma 3.1 (continuation theorem [19, page 40]). Let L be a Fredholm mapping of index zero, and let $N$ be L-compact on $\bar{\Omega}$. Suppose that

(a) for each $\lambda \in(0,1)$, every solution $x$ of $L x=\lambda N x$ is such that $x \in \partial \Omega$;

(b) $Q N x \neq 0$ for each $x \in \partial \Omega \cap \operatorname{Ker} L$ and

$$
\operatorname{deg}\{J Q N, \Omega \cap \operatorname{Ker} L, 0\} \neq 0 .
$$

Then the equation $L x=N x$ has at least one solution lying in $\operatorname{Dom} L \cap \bar{\Omega}$. 
For the simplicity of presentation, in the remaining part of this paper, for a continuous function $g:[0, \omega] \mapsto R$, we denote

$$
g^{*}=\max _{t \in[0, \omega]} g(t), \quad g_{*}=\min _{t \in[0, \omega]} g(t), \quad \bar{g}=\frac{1}{\omega} \int_{0}^{\omega} g(t) d t .
$$

Theorem 3.2. Let (A1)-(A3) hold, $k_{i j}=\left(1 / \overline{d_{i}}+\omega\right)\left(\overline{\left|a_{i j}\right|}+\overline{\left|b_{i j}\right|}+\overline{\left|c_{i j} h_{i j}\right|}\right) p_{j}$ and $K=\left(k_{i j}\right)_{n \times n}$. If $\rho(K)<1$, then system (1.1) has at least a w-periodic solution.

Proof. Take $X=Z=\left\{x(t)=\left(x_{1}(t), x_{2}(t), \ldots, x_{n}(t)\right)^{T} \in C\left(R, R^{n}\right): x(t)=x(t+\omega)\right.$, for all $\left.t \in R\right\}$, and denote

$$
\left|x_{i}\right|=\max _{t \in[0, \omega]}\left|x_{i}(t)\right|, \quad i=1,2, \ldots, n, \quad\|x\|=\max _{1 \leq i \leq n}\left|x_{i}\right| .
$$

Equipped with the norms $\|\cdot\|$, both $X$ and $Z$ are Banach spaces. Denote

$$
\begin{aligned}
\Delta\left(x_{i}, t\right):= & -d_{i}(t) x_{i}(t)+\sum_{j=1}^{n} a_{i j}(t) f_{j}\left(x_{j}(t)\right)+\sum_{j=1}^{n} b_{i j}(t) f_{j}\left(x_{j}\left(t-\tau_{i j}(t)\right)\right) \\
& +\sum_{j=1}^{n} c_{i j}(t) \int_{-\infty}^{t} H_{i j}(t-s) f_{j}\left(x_{j}(s)\right) d s+J_{i}(t) .
\end{aligned}
$$

Since

$$
\sum_{j=1}^{n} c_{i j}(t) \int_{-\infty}^{t} H_{i j}(t-s) f_{j}\left(x_{j}(s)\right) d s=\sum_{j=1}^{n} c_{i j}(t) \int_{0}^{\infty} H_{i j}(s) f_{j}\left(x_{j}(t-s)\right) d s,
$$

then, for any $x(t) \in X$, because of the periodicity, it is easy to check that

$$
\begin{aligned}
\Delta\left(x_{i}, t\right)= & -d_{i}(t) x_{i}(t)+\sum_{j=1}^{n} a_{i j}(t) f_{j}\left(x_{j}(t)\right)+\sum_{j=1}^{n} b_{i j}(t) f_{j}\left(x_{j}\left(t-\tau_{i j}(t)\right)\right) \\
& +\sum_{j=1}^{n} c_{i j}(t) \int_{0}^{\infty} H_{i j}(s) f_{j}\left(x_{j}(t-s)\right) d s+J_{i}(t) \in Z .
\end{aligned}
$$


Let

$$
\begin{aligned}
& L: \operatorname{Dom} L=\left\{x \in X: x \in C^{1}\left(R, R^{n}\right)\right\} \ni x \longmapsto x^{\prime}(\cdot) \in Z, \\
& P: X \ni x \longmapsto \frac{1}{\omega} \int_{0}^{\omega} x(t) d t \in X, \\
& Q: Z \ni z \longmapsto \frac{1}{\omega} \int_{0}^{\omega} z(t) d t \in Z, \\
& N: X \ni x \longmapsto \Delta\left(x_{i}, t\right) \in Z .
\end{aligned}
$$

Here, for any $W=\left(w_{1}, w_{2}, \ldots, w_{n}\right)^{T} \in R^{n}$, we identify it as the constant function in $X$ or $Z$ with the value vector $W=\left(w_{1}, w_{2}, \ldots, w_{n}\right)^{T}$. Then system (1.1) can be reduced to the operator equation $L x=N x$. It is easy to see that

$$
\begin{gathered}
\operatorname{Ker} L=R^{n}, \\
\operatorname{Im} L=\left\{z \in Z: \frac{1}{\omega} \int_{0}^{\omega} z(t) d t=0\right\}, \quad \text { which is closed in } Z, \\
\text { dimKer } L=\operatorname{codimIm} L=n<\infty,
\end{gathered}
$$

and $P, Q$ are continuous projectors such that

$$
\operatorname{Im} P=\operatorname{ker} L, \quad \operatorname{Ker} Q=\operatorname{Im} L=\operatorname{Im}(I-Q)
$$

It follows that $L$ is a Fredholm mapping of index zero. Furthermore, the generalized inverse (to $L$ ) $K_{p}: \operatorname{Im} L \mapsto \operatorname{Ker} P \cap \operatorname{Dom} L$ is given by

$$
\left(K_{p}(z)\right)_{i}(t)=\int_{0}^{t} z_{i}(s) d s-\frac{1}{\omega} \int_{0}^{\omega} \int_{0}^{s} z_{i}(v) d v d s
$$

Then,

$$
\begin{gathered}
(Q N x)_{i}(t)=\frac{1}{\omega} \int_{0}^{\omega} \Delta\left(x_{i}, s\right) d s \\
\left(K_{p}(I-Q) N x\right)_{i}(t)=\int_{0}^{t} \Delta\left(x_{i}, s\right) d s-\frac{1}{\omega} \int_{0}^{\omega} \int_{0}^{t} \Delta\left(x_{i}, s\right) d s d t+\left(\frac{1}{2}-\frac{t}{\omega}\right) \int_{0}^{\omega} \Delta\left(x_{i}, s\right) d s .
\end{gathered}
$$

Clearly, $Q N$ and $K_{p}(I-Q) N$ are continuous. For any bounded open subset $\Omega \subset X, Q N(\bar{\Omega})$ is obviously bounded. Moreover, applying the ArzelaCAscoli theorem, one can easily show that $\overline{K_{p}(I-Q) N(\bar{\Omega})}$ is compact. Therefore, $N$ is $L$-compact on with any bounded open subset 
$\Omega \in X$. Since $\operatorname{Im} Q=\operatorname{Ker} L$, we take the isomorphism $J$ of $\operatorname{Im} Q$ onto $\operatorname{Ker} L$ to be the identity mapping.

Now, we reach the point to search for an appropriate open bounded set $\Omega$ for the application of the continuation theorem corresponding to the operator equation $L x=\lambda N x$, $\lambda \in(0,1)$, and we have

$$
x_{i}^{\prime}(t)=\lambda \Delta\left(x_{i}, t\right) \text { for } 1=1,2, \ldots, n .
$$

Assume that $x=x(t) \in X$ is a solution of system (1.1) for some $\lambda \in(0,1)$. Integrating both sides of (3.13) over the interval $[0, \omega]$, we obtain

$$
0=\int_{0}^{\omega} x_{i}^{\prime}(t) d t=\lambda \int_{0}^{\omega} \Delta\left(x_{i}, t\right) d t
$$

Then

$$
\begin{aligned}
\int_{0}^{\omega} d_{i}(t) x_{i}(t) d t=\int_{0}^{\omega}\{ & \sum_{j=1}^{n} a_{i j}(t) f_{j}\left(x_{j}(t)\right)+\sum_{j=1}^{n} b_{i j}(t) f_{j}\left(x_{j}\left(t-\tau_{i j}(t)\right)\right) \\
& \left.+\sum_{j=1}^{n} c_{i j}(t) \int_{0}^{\infty} H_{i j}(s) f_{j}\left(x_{j}(t-s)\right) d s+J_{i}(t)\right\} d t .
\end{aligned}
$$

Noting that

$$
\left|f_{j}(u)\right| \leq p_{j}|u|+q_{j} \quad \forall u \in R, j=1,2, \ldots, n,
$$

we get

$$
\left|x_{i}\right|_{*} \overline{d_{i}} \leq \sum_{j=1}^{n}\left(\overline{\left|a_{i j}\right|}+\overline{\left|b_{i j}\right|}+\overline{\left|c_{i j} h_{i j}\right|}\right) p_{j}\left|x_{j}\right|^{*}+\sum_{j=1}^{n}\left(\overline{\left|a_{i j}\right|}+\overline{\left|b_{i j}\right|}+\overline{\left|c_{i j} h_{i j}\right|}\right) q_{j}+\overline{\left|J_{i}\right|} .
$$

It follows that

$$
\left|x_{i}\right|_{*} \leq \frac{1}{\bar{d}_{i}} \sum_{j=1}^{n}\left(\overline{\left|a_{i j}\right|}+\overline{\left|b_{i j}\right|}+\overline{\left|c_{i j} h_{i j}\right|}\right) p_{j}\left|x_{j}\right|^{*}+\frac{1}{\overline{d_{i}}}\left\{\sum_{j=1}^{n}\left(\overline{\left|a_{i j}\right|}+\overline{\left|b_{i j}\right|}+\overline{\left|c_{i j} h_{i j}\right|}\right) q_{j}+\overline{\left|J_{i}\right|}\right\} .
$$

Note that each $x_{i}(t)$ is continuously differentiable for $i=1,2, \ldots, n$, and it is certain that there exists $t_{i} \in[0, \omega]$ such that $\left|x_{i}\left(t_{i}\right)\right|=\left|x_{i}(t)\right|_{*}$. Set

$$
D=\left(D_{1}, D_{2}, \ldots, D_{n}\right)^{T}, \quad D_{i}=\left(\frac{1}{\overline{d_{i}}}+\omega\right)\left\{\sum_{j=1}^{n}\left(\overline{\left|a_{i j}\right|}+\overline{\left|b_{i j}\right|}+\overline{\left|c_{i j} h_{i j}\right|}\right) q_{j}+\overline{\left|J_{i}\right|}\right\}
$$


In view of $\rho(K)<1$ and Lemma 2.2, we have $(E-K)^{-1} D=l=\left(l_{1}, l_{2}, \ldots, l_{n}\right)^{T} \geq 0$, where $l_{i}$ is given by

$$
l_{i}=\sum_{j=1}^{n} k_{i j} l_{j}+D_{i}, \quad i=1,2, \ldots, n
$$

Let

$$
\Omega=\left\{\left(x_{1}, x_{2}, \ldots, x_{n}\right)^{T} \in R^{n} ;\left|x_{i}\right| \leq l_{i}, i=1,2, \ldots, n\right\}
$$

Then, for $t \in\left[t_{i}, t_{i}+\omega\right]$, we have

$$
\begin{aligned}
& \left|x_{i}(t)\right| \leq\left|x_{i}\left(t_{i}\right)\right|+\int_{t_{i}}^{t} D^{+}\left|x_{i}(t)\right| d t \\
& \leq\left|x_{i}(t)\right|_{*}+\int_{t_{i}}^{t_{i}+\omega} D^{+}\left|x_{i}(t)\right| d t \\
& \leq \frac{1}{\bar{d}_{i}} \sum_{j=1}^{n}\left(\overline{\left|a_{i j}\right|}+\overline{\left|b_{i j}\right|}+\overline{\left|c_{i j} h_{i j}\right|}\right) p_{j}\left|x_{j}\right|^{*} \\
& +\frac{1}{\overline{d_{i}}}\left\{\sum_{j=1}^{n}\left(\overline{\left|a_{i j}\right|}+\overline{\left|b_{i j}\right|}+\overline{\left|c_{i j} h_{i j}\right|}\right) q_{j}+\overline{\left|J_{i}\right|}\right\}+\int_{t_{i}}^{t_{i}+\omega} D^{+}\left|x_{i}(t)\right| d t \\
& \leq\left(\frac{1}{\overline{d_{i}}}+\omega\right) \sum_{j=1}^{n}\left(\overline{\left|a_{i j}\right|}+\overline{\left|b_{i j}\right|}+\overline{\left|c_{i j} h_{i j}\right|}\right) p_{j}\left|x_{j}\right|^{*} \\
& +\left(\frac{1}{\overline{d_{i}}}+\omega\right)\left\{\sum_{j=1}^{n}\left(\overline{\left|a_{i j}\right|}+\overline{\left|b_{i j}\right|}+\overline{\left|c_{i j} h_{i j}\right|}\right) q_{j}+\overline{\left|J_{i}\right|}\right\} \\
& \leq \sum_{j=1}^{n} k_{i j} l_{j}+D_{i} \\
& =l_{i} \text {, }
\end{aligned}
$$

where $D^{+}$denotes the right derivative. Clearly, $l_{i}, i=1,2, \ldots, n$, are independent of $\lambda$. Then there are no $\lambda \in(0,1)$ and $x \in \Omega$ such that $L x=\lambda N x$. When $u=\left(x_{1}, x_{2}, \ldots, x_{n}\right)^{T} \in \partial \Omega \cap$ $\operatorname{Ker} L=\partial \Omega \cap R^{n}, u$ is a constant vector in $R^{n}$ with $\left|x_{i}\right|=l_{i}, i=1,2, \ldots, n$. Note that $Q N u=$ $J Q N u$; when $u \in \operatorname{Ker} L$, it must be

$$
(Q N u)_{i}=-\overline{d_{i}}+\sum_{j=1}^{n}\left(\overline{a_{i j}}+\overline{b_{i j}}+\overline{c_{i j} h_{i j}}\right) f_{j}\left(x_{j}\right)+\overline{J_{i}} .
$$


We claim that

$$
\left|(Q N u)_{i}\right|>0 \text { for } i=1,2, \ldots, n \text {. }
$$

On the contrary, suppose that there exists some $i$ such that $\left|(Q N u)_{i}\right|=0$, that is,

$$
\overline{d_{i}} x_{i}=\sum_{j=1}^{n}\left(\overline{a_{i j}}+\overline{b_{i j}}+\overline{c_{i j} h_{i j}}\right) f_{j}\left(x_{j}\right)+\overline{J_{i}} \text {. }
$$

Then, we have

$$
\begin{aligned}
& l_{i}=\left|x_{i}\right| \\
& =\frac{1}{\overline{d_{i}}}\left|\sum_{j=1}^{n}\left(\overline{a_{i j}}+\overline{b_{i j}}+\overline{c_{i j} h_{i j}}\right) f_{j}\left(x_{j}\right)+\overline{J_{i}}\right| \\
& \leq \frac{1}{\bar{d}_{i}} \sum_{j=1}^{n}\left(\overline{\left|a_{i j}\right|}+\overline{\left|b_{i j}\right|}+\overline{\left|c_{i j} h_{i j}\right|}\right) p_{j} l_{j} \\
& +\frac{1}{\overline{d_{i}}}\left\{\sum_{j=1}^{n}\left(\overline{\left|a_{i j}\right|}+\overline{\left|b_{i j}\right|}+\overline{\left|c_{i j} h_{i j}\right|}\right) q_{j}+\overline{\left|J_{i}\right|}\right\} \\
& \leq\left(\frac{1}{\bar{d}_{i}}+\omega\right) \sum_{j=1}^{n}\left(\overline{\left|a_{i j}\right|}+\overline{\left|b_{i j}\right|}+\overline{\left|c_{i j} h_{i j}\right|}\right) p_{j} l_{j} \\
& +\left(\frac{1}{\bar{d}_{i}}+\omega\right)\left\{\sum_{j=1}^{n}\left(\overline{\left|a_{i j}\right|}+\overline{\left|b_{i j}\right|}+\overline{\left|c_{i j} h_{i j}\right|}\right) q_{j}+\overline{\left|J_{i}\right|}\right\} \\
& =\sum_{j=1}^{n} k_{i j} l_{j}+D_{i} \\
& =l_{i} \text {, }
\end{aligned}
$$

which is a contradiction. Therefore,

$$
Q N u \neq 0 \quad \text { for any } u \in \partial \Omega \cap \operatorname{Ker} L=\partial \Omega \cap R^{n} \text {. }
$$

Consider the homotopy $F:(\Omega \cap \operatorname{Ker} L) \times[0,1] \mapsto \Omega \cap \operatorname{Ker} L$ defined by

$$
F(u, \mu)=\mu \operatorname{diag}\left(-\overline{d_{1}},-\overline{d_{2}}, \ldots,-\overline{d_{n}}\right) u+(1-\mu) Q N u,
$$


$(u, \mu) \in(\Omega \cap \operatorname{Ker} L) \times[0,1]$. Note that $F(\cdot, 0)=J Q N$; if $F(u, \mu)=0$, then, as before, we have

$$
\begin{aligned}
\left|x_{i}\right|= & \frac{1-\mu}{\overline{d_{i}}}\left|\sum_{j=1}^{n}\left(\overline{a_{i j}}+\overline{b_{i j}}+\overline{c_{i j} h_{i j}}\right) f_{j}\left(x_{j}\right)+\overline{J_{i}}\right| \\
\leq & \frac{1}{\bar{d}_{i}} \sum_{j=1}^{n}\left(\overline{\left|a_{i j}\right|}+\overline{\left|b_{i j}\right|}+\overline{\left|c_{i j} h_{i j}\right|}\right) p_{j}\left|x_{j}\right| \\
& +\frac{1}{\overline{d_{i}}}\left\{\sum_{j=1}^{n}\left(\overline{\left|a_{i j}\right|}+\overline{\left|b_{i j}\right|}+\overline{\left|c_{i j} h_{i j}\right|}\right) q_{j}+\overline{\left|J_{i}\right|}\right\} \\
\leq & \frac{1}{\overline{d_{i}}} \sum_{j=1}^{n}\left(\overline{\left|a_{i j}\right|}+\overline{\left|b_{i j}\right|}+\overline{\left|c_{i j} h_{i j}\right|}\right) p_{j} l_{j} \\
& +\frac{1}{\overline{d_{i}}}\left\{\sum_{j=1}^{n}\left(\overline{\left|a_{i j}\right|}+\overline{\left|b_{i j}\right|}+\overline{\left|c_{i j} h_{i j}\right|}\right) q_{j}+\overline{\left|J_{i}\right|}\right\} \\
< & \sum_{j=1}^{n} k_{i j} l_{j}+D_{i} \\
= & l_{i}
\end{aligned}
$$

Hence

$$
F(u, \mu) \neq 0, \quad \text { for }(u, \mu) \in(\partial \Omega \cap \operatorname{Ker} L) \times[0,1] .
$$

It follows from the property of invariance under a homotopy that

$$
\begin{aligned}
\operatorname{deg}\{J Q N, \Omega \cap \operatorname{Ker} L, 0\} & =\operatorname{deg}\{F(\cdot, 0), \Omega \cap \operatorname{Ker} L, 0\} \\
& =\operatorname{deg}\{F(\cdot, 1), \Omega \cap \operatorname{Ker} L, 0\}=\operatorname{deg}\left\{\operatorname{diag}\left(-\overline{d_{1}},-\overline{d_{2}}, \ldots,-\overline{d_{n}}\right)\right\} \neq 0 .
\end{aligned}
$$

Thus, we have shown that $\Omega$ satisfies all the assumptions of Lemma 3.1. Hence, $L u=N u$ has at least one $\omega$-periodic solution on Dom $L \cap \bar{\Omega}$. This completes the proof.

When $c_{i j}=0,(1.1)$ turns into the following system:

$$
x_{i}^{\prime}(t)=-d_{i}(t) x_{i}(t)+\sum_{j=1}^{n} a_{i j}(t) f_{j}\left(x_{j}(t)\right)+\sum_{j=1}^{n} b_{i j}(t) f_{j}\left(x_{j}(t-\tau(t))\right)+J_{i}(t), \quad i=1,2, \ldots, n
$$

Corollary 3.3. Let (A1)-(A3) hold, $k_{i j}=\left(1 / \overline{d_{i}}+\omega\right)\left(\overline{\left|a_{i j}\right|}+\overline{\left|b_{i j}\right|}\right) p_{j}$, and $K=\left(k_{i j}\right)_{n \times n}$. If $\rho(K)<1$, then system $(P)$ has at least a $\omega$-periodic solution. 
Theorem 3.4. Let (A1), (A2), and (A4) hold, $k_{i j}=\left(1 / \overline{d_{i}}+\omega\right)\left(\overline{\left|a_{i j}\right|}+\overline{\left|b_{i j}\right|}+\overline{\left|c_{i j} h_{i j}\right|}\right) p_{j}$, and $K=$ $\left(k_{i j}\right)_{n \times n}$. If $\rho(K)<1$, and that

$$
\overline{d_{i}}-\sum_{j=1}^{n}\left(\overline{\left|a_{i j}\right|}+\overline{\left|b_{i j}\right|}+\overline{\left|c_{i j} h_{i j}\right|}\right) p_{j} e^{d_{i}^{*} \tau}>0,
$$

then system (1.1) has exactly one w-periodic solution. Moreover, it is globally exponentially stable.

Proof. Let $C=C\left([-\tau, 0], R^{n}\right)$ with the supnorm $\|\varphi\|=\sup _{s \in[-\tau, 0] ; 1 \leq i \leq n}\left|\varphi_{i}(s)\right|, \varphi \in C$. As usual, if $(-\infty \leq) a \leq b(\leq \infty)$ and $\psi \in C\left([-\tau+a, b], R^{n}\right)$, then for $t \in[a, b]$ we define $\psi_{t} \in C$ by $\psi_{t}(\theta)=$ $\psi(t+\theta), \theta \in[-\tau, 0]$. From (A4), we can get $\left|f_{j}(u)\right| \leq p_{j}|u|+\left|f_{j}(0)\right|, j=1,2, \ldots, n$. Hence, all the hypotheses in Theorem 3.2 hold with $q_{j}=\left|f_{j}(0)\right|, j=1,2, \ldots, n$. Thus, system (1.1) has at least one $\omega$-periodic solution, say $\tilde{x}(t)=\left(\tilde{x}_{1}(t), \tilde{x}_{2}(t), \ldots, \tilde{x}_{n}(t)\right)^{T}$. Let $x(t)=\left(x_{1}(t), x_{2}(t), \ldots, x_{n}(t)\right)^{T}$ be an arbitrary solution of system (1.1). For $t \geq 0$, a direct calculation of the right derivative $D^{+}\left|x_{i}(t)-\tilde{x}_{i}(t)\right|$ of $\left|x_{i}(t)-\tilde{x}_{i}(t)\right|$ along the solutions of system (1.1) leads to

$$
\begin{aligned}
D^{+}\left|x_{i}(t)-\tilde{x}_{i}(t)\right|= & D^{+}\left\{\operatorname{sgn}\left(x_{i}(t)-\tilde{x}_{i}(t)\right\}\left(x_{i}(t)-\tilde{x}_{i}(t)\right)\right. \\
\leq & -d_{i}(t)\left|x_{i}(t)-\tilde{x}_{i}(t)\right|+\sum_{j=1}^{n}\left(\left|a_{i j}(t)\left[f_{j}\left(x_{j}(t)\right)-f_{j}\left(\tilde{x}_{j}(t)\right)\right]\right|\right. \\
& +\sum_{j=1}^{n}\left|b_{i j}(t)\left[f_{j}\left(x_{j}\left(t-\tau_{i j}(t)\right)\right)-f_{j}\left(\tilde{x}_{j}\left(t-\tau_{i j}(t)\right)\right)\right]\right| \\
& +\sum_{j=1}^{n}\left|c_{i j}(t)\right|\left|\int_{0}^{+\infty} k_{i j}(s)\left[f_{j}\left(x_{j}(t-s)\right)-f_{j}\left(\tilde{x}_{j}(t-s)\right)\right] d s\right| \\
\leq & -d_{i}(t)\left|x_{i}(t)-\tilde{x}_{i}(t)\right|+\sum_{j=1}^{n}\left|a_{i j}(t)\right| p_{j}\left|x_{j}(t)-\tilde{x}_{j}(t)\right| \\
& +\sum_{j=1}^{n}\left|b_{i j}(t)\right| p_{j}\left|x_{j}\left(t-\tau_{i j}(t)\right)-\tilde{x}_{j}\left(t-\tau_{i j}(t)\right)\right| \\
& +\sum_{j=1}^{n}\left|c_{i j}(t) h_{i j}\right| p_{j} \sup _{-\tau \leq s \leq t}\left|x_{j}(s)-\tilde{x}_{j}(s)\right| \\
\leq & -d_{i}(t)\left|x_{i}(t)-\tilde{x}_{i}(t)\right| \\
& +\sum_{j=1}^{n}\left(\left|a_{i j}(t)\right|+\left|b_{i j}(t)\right|+\left|c_{i j}(t) h_{i j}\right|\right) p_{j} \sup _{-\tau \leq s \leq t}\left|x_{j}(s)-\tilde{x}_{j}(t)\right| .
\end{aligned}
$$

Let $z_{i}(t)=\left|x_{i}(t)-\tilde{x}_{i}(t)\right|$. Then (3.33) can be transformed into

$$
D^{+} z_{i}(t) \leq-d_{i}(t) z_{i}(t)+\sum_{j=1}^{n}\left(\left|a_{i j}(t)\right|+\left|b_{i j}(t)\right|+\left|c_{i j}(t) h_{i j}\right|\right) p_{j} \sup _{-\tau \leq s \leq t} z_{j}(s) .
$$


Thus, for $t>t_{0}$ we have

$$
D^{+}\left\{z_{i}(t) e^{\int_{t_{0}}^{t} d_{i}(s) d s}\right\} \leq \sum_{j=1}^{n}\left(\left|a_{i j}(t)\right|+\left|b_{i j}(t)\right|+\left|c_{i j}(t) h_{i j}\right|\right) p_{j}\left\|z_{t}\right\| e^{\int_{t_{0}}^{t} d_{i}(s) d s},
$$

It follows that

$$
\begin{aligned}
z_{i}(t) e^{\int_{t_{0}}^{t} d_{i}(s) d s} \leq & \left|z_{i}\left(t_{0}\right)\right| \\
& +\int_{t_{0}}^{t}\left\{\sum_{j=1}^{n}\left(\left|a_{i j}(u)\right|+\left|b_{i j}(u)\right|+\left|c_{i j}(u) h_{i j}\right|\right) p_{j}\left\|z_{u}\right\| e^{\int_{t_{0}}^{u} d_{i}(s) d s}\right\} d u .
\end{aligned}
$$

Thus, for any $t>0$ and $\theta \in[-\min (\tau, t), 0]$, we have

$$
e^{\int_{t_{0}}^{t+\theta} d_{i}(s) d s}=e^{\left(\int_{t_{0}}^{t}+\int_{t}^{t+\theta}\right) d_{i}(s) d s} \geq e^{\int_{t_{0}}^{t} d_{i}(s) d s-d_{i}^{*} \tau}
$$

Therefore,

$$
\begin{aligned}
e^{\int_{t_{0}}^{t} d_{i}(s) d s-d_{i}^{*} \tau} z_{i}(t+\theta) & \leq e^{\int_{t_{0}}^{t+\theta} d_{i}(s) d s} z_{i}(t+\theta) \\
& \leq\left\|z_{t_{0}}\right\|+\int_{t_{0}}^{t+\theta}\left\{\sum_{j=1}^{n}\left(\left|a_{i j}(u)\right|+\left|b_{i j}(u)\right|+\left|c_{i j}(u) h_{i j}\right|\right) p_{j}\left\|z_{u}\right\| e^{\int_{t_{0}}^{u} d_{i}(s) d s}\right\} d u .
\end{aligned}
$$

It follows that

$$
\begin{aligned}
e^{\int_{t_{0}}^{t} d_{i}(s) d s}\left\|z_{t}\right\| \leq & e^{d_{i}^{*} \tau}\left\|z_{t_{0}}\right\| \\
& +\int_{t_{0}}^{t} e^{d_{i}^{*} \tau}\left\{\sum_{j=1}^{n}\left(\left|a_{i j}(u)\right|+\left|b_{i j}(u)\right|+\left|c_{i j}(u) h_{i j}\right|\right) p_{j}\left\|z_{u}\right\| e^{\int_{t_{0}}^{u} d_{i}(s) d s}\right\} d u .
\end{aligned}
$$

By Gronwall's inequality, we obtain

$$
\left\|z_{t}\right\| \leq e^{d_{i}^{*} \tau}\left\|z_{t_{0}}\right\| e^{\int_{t_{0}}^{t} e^{d_{i}^{*} \tau} \sum_{j=1}^{n}\left(\left|a_{i j}(u)\right|+\left|b_{i j}(u)\right|+\left|c_{i j}(u) h_{i j}\right|\right) p_{j} d u} e^{\int_{t_{0}}^{t}-d_{i}(s) d s}, \quad t \geq t_{0} .
$$


Without loss of generality, we let $t_{0}=0$. For $t \geq 0,[t / \omega]$ denotes the largest integer less than or equal to $t / \omega$. Noting $[t / \omega] \geq t / \omega-1$, and $\overline{d_{i}}>\sum_{j=1}^{n}\left(\left|\overline{a_{i j}}\right|+\left|\overline{b_{i j}}\right|+\left|\overline{c_{i j}} h_{i j}\right|\right) p_{j} e^{d_{i}^{*} \tau}$, we get

$$
\begin{aligned}
& \left\|z_{t}\right\| \leq e^{d_{i}^{*} \tau}\left\|z_{0}\right\| e^{\int_{0}^{t} e^{d_{i}^{*} \tau} \sum_{j=1}^{n}\left(\left|a_{i j}(u)\right|+\left|b_{i j}(u)\right|+\left|c_{i j}(u) h_{i j}\right|\right) p_{j} d u} e^{\int_{0}^{t}-d_{i}(s) d s} \\
& =e^{d_{i}^{*} \tau}\left\|z_{0}\right\| e^{\left(\int_{0}^{\omega[t / \omega]}+\int_{\omega[t / \omega]}^{t}\right)\left\{e^{d_{i}^{*} \tau} \sum_{j=1}^{n}\left(\left|a_{i j}(u)\right|+\left|b_{i j}(u)\right|+\left|c_{i j}(u) h_{i j}\right|\right) p_{j}-d_{i}(u)\right\} d u} \\
& \leq e^{d_{i}^{*} \tau+\left(-\overline{d_{i}}+\sum_{j=1}^{n}\left(\overline{a_{i j} \mid}|+| \overline{b_{i j}}|+| \overline{c_{i j} h_{i j} \mid} \mid\right) p_{j} e^{d_{i}^{*} \tau}\right) \omega[t / \omega]}
\end{aligned}
$$

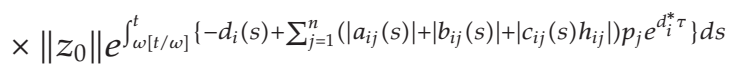

$$
\begin{aligned}
& \left.\leq e^{d_{i}^{*} \tau+\left(-\overline{d_{i}}+\sum_{j=1}^{n}\left(\overline{a_{i j} \mid}|+| \overline{b_{i j}}|+| \overline{c_{i j}} h_{i j} \mid\right)\right.} p_{j} e^{d_{i}^{*} \tau}\right) \omega[t / \omega]
\end{aligned}
$$

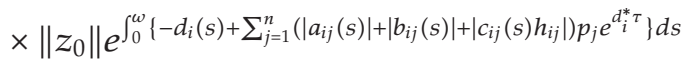

$$
\begin{aligned}
& \leq e^{d_{i}^{*} \tau}\left\|z_{0}\right\| e^{-\left\{\overline{d_{i}}-\sum_{j=1}^{n}\left(\overline{a_{i j} \mid}+\overline{b_{i j} \mid}+\overline{c_{i j} h_{i j} \mid}\right) p_{j} e^{d_{i}^{*} \tau}\right\} t} \\
& \leq m\left\|z_{0}\right\| e^{-\lambda t}, \quad t \geq 0,
\end{aligned}
$$

where $m=\max _{1 \leq i \leq n}\left\{e^{d_{i}^{*} \tau}\right\}$ and $\lambda=\min _{1 \leq i \leq n}\left\{\overline{d_{i}}-\sum_{j=1}^{n}\left(\overline{\left|a_{i j}\right|}+\overline{\left|b_{i j}\right|}+\overline{\left|c_{i j} h_{i j}\right|}\right) p_{j} e^{d_{i}^{*} \tau}\right\}$ are positive constants. From (3.41), it is obvious that the periodic solution is global exponentially stable, and this completes the proof of Theorem 3.4.

Corollary 3.5. Let (A1), (A2), and (A4) hold, $k_{i j}=\left(1 / \overline{d_{i}}+\omega\right)\left(\overline{\left|a_{i j}\right|}+\overline{\left|b_{i j}\right|}\right) p_{j}$ and $K=\left(k_{i j}\right)_{n \times n}$. If $\rho(K)<1$, and that

$$
\overline{d_{i}}-\sum_{j=1}^{n}\left(\overline{\left|a_{i j}\right|}+\overline{\left|b_{i j}\right|}\right) p_{j} e^{d_{i}^{*} \tau}>0
$$

then system $(P)$ has exactly one w-periodic solution. Moreover, it is globally exponentially stable.

Remark 3.6. To the best of our knowledge, few authors have considered the existence of periodic solution and global exponential stability for model (1.1) with coefficients and delays all periodically varying in time. We only find model $(P)$ in $[20,21]$; however, it is assumed in [20] that $\tau_{i j}(t) \geq 0$ are constants and in [21] that $a_{i j}(t), b_{i j}(t), J_{i}(t)$ are continuous $\omega$ periodic functions, and $d_{i}$ are positive constants. Especially, the authors of [21] suppose that $\tau_{i j}(t) \geq 0$ are continuously differentiable $\omega$-periodic functions and $0 \leq \tau_{i j}^{\prime}(t)<1$, clearly, which implies that $\tau_{i j}(t)$ are also constants. Obviously, our model is more general. Furthermore, in $[20,21] f_{i}, i=1,2, \ldots, n$, are assumed to be strictly monotone, and the explicit presence of the maximum value of the coefficients functions in Theorems 3.2 and 3.4 (see $[20,21])$ may impose a very strict constraint on the model (e.g., when some of the maximum value of the coefficients functions are very large). Therefore, our results are more convenient when designing a cellular neural network. 


\section{An Example}

In this section, an example is used to demonstrate that the method presented in this paper is effective.

Example 4.1. Consider the following two state neural networks:

$$
\begin{aligned}
\left(\begin{array}{l}
x_{1}^{\prime}(t) \\
x_{2}^{\prime}(t)
\end{array}\right)= & -\left(\begin{array}{cc}
d_{1}(t) & 0 \\
0 & d_{2}(t)
\end{array}\right)\left(\begin{array}{l}
x_{1}(t) \\
x_{2}(t)
\end{array}\right)+\left(\begin{array}{ll}
a_{11}(t) & a_{12}(t) \\
a_{21}(t) & a_{22}(t)
\end{array}\right)\left(\begin{array}{l}
f_{1}\left(x_{1}(t)\right) \\
f_{2}\left(x_{2}(t)\right)
\end{array}\right) \\
& +\left(\begin{array}{ll}
b_{11}(t) & b_{12}(t) \\
b_{21}(t) & b_{22}(t)
\end{array}\right)\left(\begin{array}{l}
f_{1}\left(x_{1}\left(t-\tau_{1}(t)\right)\right) \\
f_{2}\left(x_{2}\left(t-\tau_{2}(t)\right)\right)
\end{array}\right) \\
& +\left(\begin{array}{cc}
c_{11}(t) & c_{12}(t) \\
c_{21}(t) & c_{22}(t)
\end{array}\right) \int_{-\infty}^{t}\left(\begin{array}{ll}
H_{11}(t-s) & H_{12}(t-s) \\
H_{21}(t-s) & H_{22}(t-s)
\end{array}\right)\left(\begin{array}{l}
f_{1}\left(x_{1}(s)\right) \\
f_{2}\left(x_{2}(s)\right)
\end{array}\right) d s \\
& +\left(\begin{array}{c}
3 \cos t \\
2 \sin t
\end{array}\right)
\end{aligned}
$$

where, all $d_{i}(t)>0, a_{i j}(t), b_{i j}(t), c_{i j}(t), \tau_{i}(t)$ are $2 \pi$-periodic continuous functions. The activation function $f_{1}(x)=\cos ((1 / 3) x)+(1 / 3) x, f_{2}(x)=\sin ((1 / 2) x)+(1 / 4) x . \tau=0.6, \overline{d_{1}}=4$, $\overline{d_{2}}=3 ; \overline{\left|a_{11}\right|}+\overline{\left|b_{11}\right|}+\overline{\left|c_{11} h_{11}\right|}=3 / 80 ; \overline{\left|a_{12}\right|}+\overline{\left|b_{12}\right|}+\overline{\left|c_{12} h_{12}\right|}=1 / 6 ; \overline{\left|a_{21}\right|}+\overline{\left|b_{21}\right|}+\overline{\left|c_{21} h_{21}\right|}=3 / 40$; $\overline{\left|a_{22}\right|}+\overline{\left|b_{22}\right|}+\overline{\left|c_{22} h_{22}\right|}=2 / 21 ; d_{1}^{*}=5, d_{2}^{*}=4$. Clearly, $f_{i}$ satisfies the hypothesis with $p_{1}=2 / 3$, $p_{2}=3 / 4$. By some simple calculations, we have

$$
\begin{gathered}
\overline{d_{i}}-\sum_{j=1}^{n}\left(\overline{\left|a_{i j}\right|}+\overline{\left|b_{i j}\right|}+\overline{\left|c_{i j} h_{i j}\right|}\right) p_{j} e^{d_{i}^{*} \tau}>0, \quad i=1,2, \\
K=\left(\begin{array}{ll}
\frac{1+8 \pi}{160} & \frac{1+8 \pi}{32} \\
\frac{1+6 \pi}{60} & \frac{1+6 \pi}{42}
\end{array}\right), \quad \rho(K) \approx 0.860<1 .
\end{gathered}
$$

Therefore, by Theorem 3.4, the system (1.1) has an exponentially stable $2 \pi$-periodic solution.

\section{Acknowledgment}

The first author was partially supported financially by the National Natural Science Foundation of China (10801088).

\section{References}

[1] S. Arik, "Global robust stability of delayed neural networks," IEEE Transactions on Circuits and Systems I, vol. 50, no. 1, pp. 156-160, 2003.

[2] M. Dong, "Global exponential stability and existence of periodic solutions of CNNs with delays," Physics Letters A, vol. 300, no. 1, pp. 49-57, 2002. 
[3] A. Chen, J. Cao, and L. Huang, "Global robust stability of interval cellular neural networks with time-varying delays," Chaos, Solitons E Fractals, vol. 23, no. 3, pp. 787-799, 2005.

[4] J. Cao and M. Dong, "Exponential stability of delayed bi-directional associative memory networks," Applied Mathematics and Computation, vol. 135, no. 1, pp. 105-112, 2003.

[5] T.-L. Liao and F.-C. Wang, "Global stability for cellular neural networks with time delay," IEEE Transactions on Neural Networks, vol. 11, no. 6, pp. 1481-1484, 2000.

[6] Y. Li, "Global exponential stability of BAM neural networks with delays and impulses," Chaos, Solitons E Fractals, vol. 24, no. 1, pp. 279-285, 2005.

[7] J. Cao, "On exponential stability and periodic solutions of CNNs with delays," Physics Letters A, vol. 267, no. 5-6, pp. 312-318, 2000.

[8] X. Liao and J. Wang, "Global dissipativity of continuous-time recurrent neural networks with time delay," Physical Review E, vol. 68, no. 1, Article ID 016118, 7 pages, 2003.

[9] H. Jiang and Z. Teng, "Global eponential stability of cellular neural networks with time-varying coefficients and delays," Neural Networks, vol. 17, no. 10, pp. 1415-1425, 2004.

[10] S. Arik, "An analysis of global asymptotic stability of delayed cellular neural networks," IEEE Transactions on Neural Networks, vol. 13, no. 5, pp. 1239-1242, 2002.

[11] L. O. Chua and L. Yang, "Cellular neural networks: theory," IEEE Transactions on Circuits and Systems, vol. 35, no. 10, pp. 1257-1272, 1988.

[12] T.-L. Liao and F.-C. Wang, "Global stability for cellular neural networks with time delay," IEEE Transactions on Neural Networks, vol. 11, no. 6, pp. 1481-1484, 2000.

[13] S Arik, "Stability analysis of delayed neural networks," IEEE Transactions on Circuits and Systems I, vol. 47, no. 7, pp. 1089-1092, 2000.

[14] L. Huang, C. Huang, and B. Liu, "Dynamics of a class of cellular neural networks with time-varying delays," Physics Letters A, vol. 345, no. 4-6, pp. 330-344, 2005.

[15] S. Mohamad and K. Gopalsamy, "Exponential stability of continuous-time and discrete-time cellular neural networks with delays," Applied Mathematics and Computation, vol. 135, no. 1, pp. 17-38, 2003.

[16] X. Lou and B. Cui, "Global asymptotic stability of delay BAM neural networks with impulses based on matrix theory," Applied Mathematical Modelling, vol. 32, no. 2, pp. 232-239, 2008.

[17] J. P. LaSalle, The Stability of Dynamical Systems, SIAM, Philadelphia, Pa, USA, 1976.

[18] K. Deimling, Nonlinear Functional Analysis, Springer, Berlin, Germany, 1985.

[19] R. E. Gaines and J. L. Mawhin, Coincidence Degree, and Nonlinear Differential Equations, vol. 568 of Lecture Notes in Mathematics, Springer, Berlin, Germany, 1977.

[20] J. Zhou, Z. Liu, and G. Chen, "Dynamics of periodic delayed neural networks," Neural Networks, vol. 17 , no. 1, pp. 87-101, 2004.

[21] Z. Liu and L. Liao, "Existence and global exponential stability of periodic solution of cellular neural networks with time-varying delays," Journal of Mathematical Analysis and Applications, vol. 290, no. 1, pp. 247-262, 2004. 


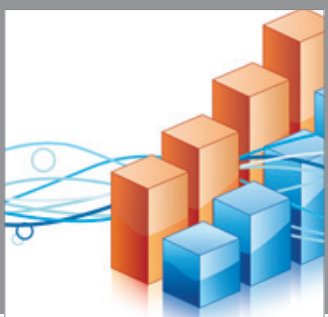

Advances in

Operations Research

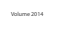

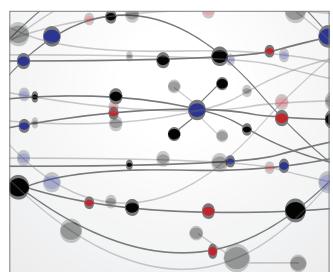

\section{The Scientific} World Journal
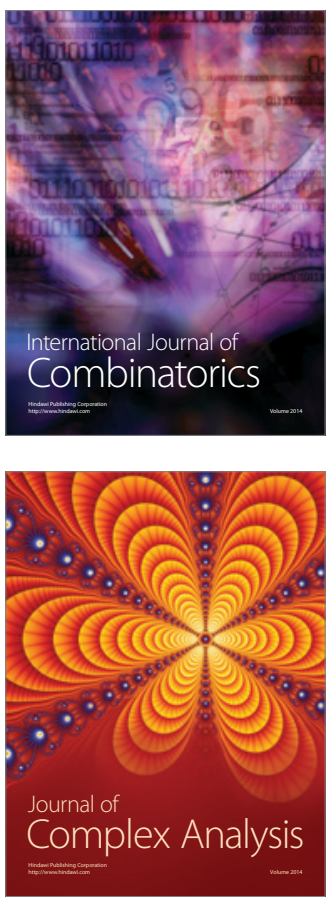

International Journal of

Mathematics and

Mathematical

Sciences
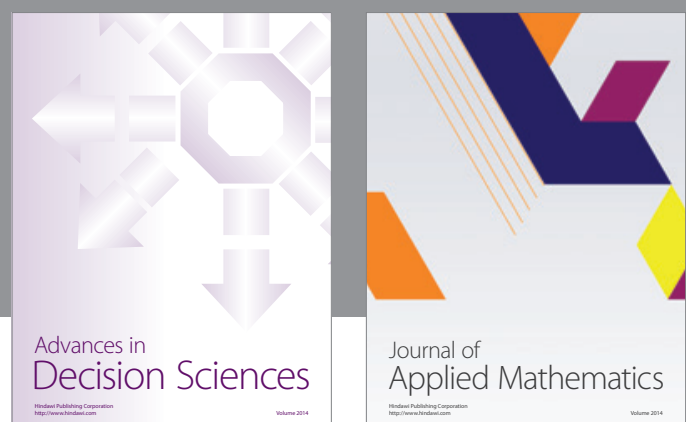

Journal of

Applied Mathematics
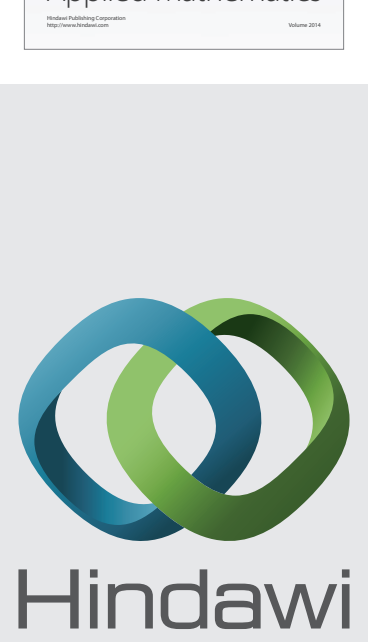

Submit your manuscripts at http://www.hindawi.com
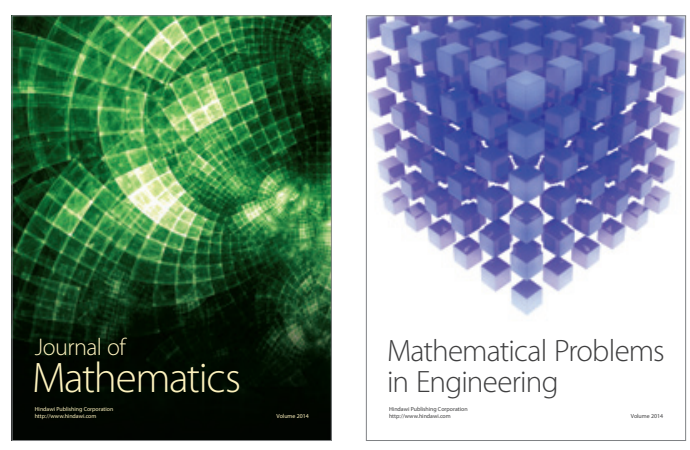

Mathematical Problems in Engineering
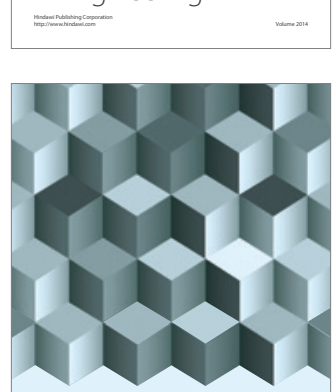

Journal of

Function Spaces
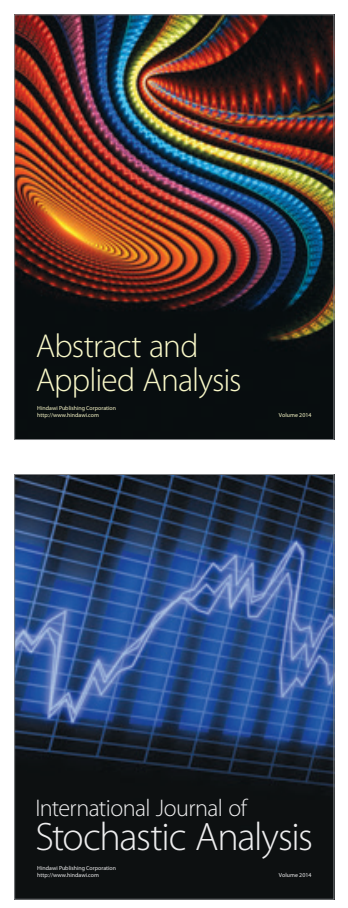

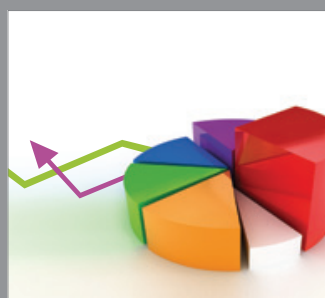

ournal of

Probability and Statistics

Promensencen
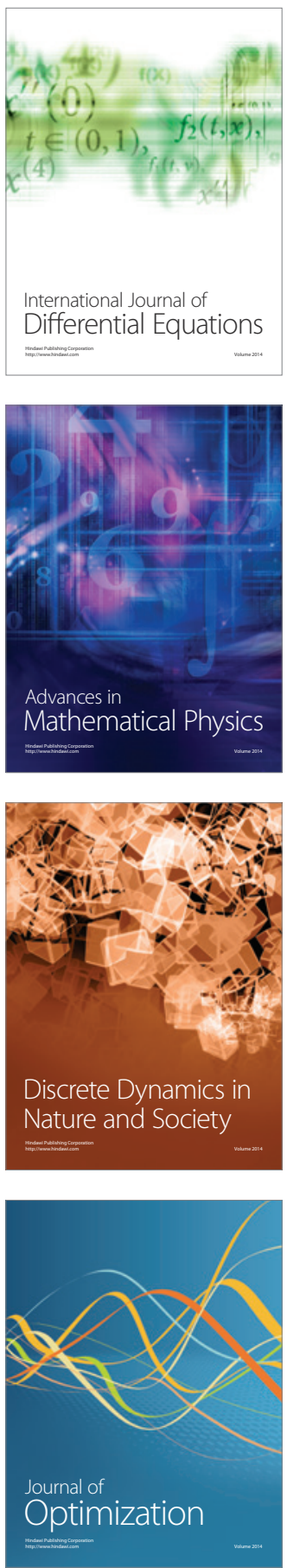